\title{
A study on the response of 2D ion chamber array detector for VMAT delivery
}

\begin{abstract}
Purpose: This study aims to evaluate the response of 2D ion chamber array detector for VMAT delivery.

Methods: 2D array ionization chamber equipped with 729 ionization chambers uniformly arranged in a $27 \times 27$ matrix with an active $27 \times 27 \mathrm{~cm}^{2}$ was used for the study. An Octagonal shaped phantom (Octavius Phantom) with a central cavity is used to insert the 2D ion chamber array. All measurements were done with a linear accelerator. The plans that are already being verified has been selected for studying the response of $2 \mathrm{D}$ array.
\end{abstract}

Results: This study shows that the response of the 2D array gets reduced as compared to early quality assured plans. Plans were analyzed using the Gamma analysis method. The results show a variation up to $2 \%$ comparing earlier plans.

Conclusion: The response of the 2D array gets reduced while compared with earlier quality assured plans.

Keywords: VMAT, 2D array, Octavius phantom, gamma analysis

\section{Introduction}

Volumetric intensity modulated single arc technique originally investigated by Otto. The concept of rotational intensity-modulated radiation therapy was first described by Rock Mackie in (1993). It will be commissioned with dosimetric accuracy and also we can favorable with other methods of IMRT delivery. VMAT represents a new paradigm in the treatment of patients with external beam radiotherapy. It uses one or more gantry arcs to deliver dose either by coplanar or non-coplanar beams. A shorter treatment time is a significant improvement for both patient compliance issues, due to confining immobilization and respiratory control devices, and patient throughput. The beams are dynamic in orientation and aperture shape and may include small apertures. The scatter conditions are expected to differ in such beams, compared to classical open fields. So the calibration carried out under broad-beam conditions may not be relevant for VMAT. ${ }^{1}$

VMAT consists of treating the patient by means of one or more gantry arcs with continuously varying beam aperture, gantry speed and dose-rate. This approach promises to maximize the benefit of intensitymodulated radiation therapy (IMRT) by treating the patient with the widest range of beam orientations. VMAT is similar to tomotherapy in that the linac source rotates through a $360^{\circ}$ arc around the patient. It is very difficult to formally analyse the behavior of VMAT because the source is continuously in motion. Both the multiple-static-field (MSF) MLC IMRT delivery method and the dynamic MLC(dMLC) IMRT delivery method assume the source to be static and a series of the modulated fields are built up from such fixed-gantry-orientation positions(see Webb 1997 for review). ${ }^{2-5}$

This technique is characterized by the simultaneous movement of the multi leaf collimator (MLC) and number of MU per degree of gantry rotation is restricted during optimization so that gantry rotation speed, leaf translational speed and dose rate maxima do not excessively limit the delivery efficiency. The gantry angle and MLC movements will be as low as $1 \mathrm{deg}$ and $0.5 \mathrm{~cm}$ is too desirable for accurate dose modeling. The gantry speed and dose rate can vary differentiating the VMAT delivery from the Intensity-modulated
Volume 5 Issue 4 - 2018

\section{SA Syam Kumar,Anu George \\ Department of Radiation Oncology, Division of Radiation Physics, India}

Correspondence: SA Syam Kumar, Department of Radiation Oncology, Division of Radiation Physics, Malabar Cancer Centre, Thalassery, Kerala, India,Tel +9| 996|443954, Email syam.ppm@gmail.com

Received: May 27, 2018 | Published: August 07, 2018 radiotherapy (IMRT). Then the fluence rate and gantry speed are kept constant. The MLC movement variable dose rates and gantry speeds of a Varian can be controlled precisely for accurate rapid arc delivery. VMAT delivery depend not only the beam fluence, but also on the aperture width, leaf, backup diaphragm and X-diaphragm position as well as their synchronization. ${ }^{6,7}$

This project aims to evaluate the response of 2D seven29 ion chamber array by delivering different VMAT plans. For this study 45 cases that are already being treated with VMAT was selected, which include QA plans that were delivered 2 years back, 1.5 years and one year back. The present gamma values were compared with the previous ones. ${ }^{8}$

\section{Materials and methods}

\section{D array}

PTW729 Freiburg Germany is made up of 729 air vented cubic ionization chamber of $5 \mathrm{~mm} \times 5 \mathrm{~mm} \times 5 \mathrm{~mm}$ each with a center to center spacing of $1 \mathrm{~cm}$, is used for measuring dose generated in a plane by a radiation beam. Wall material is made up of Graphite. The material is surrounded the vented ionization chamber is Poly Methyl Metha Acrylate (PMMA). It is arranged uniformly in a $27 \times 27$ matrix. Central axis of radiation passing through $14 \times 14$ chamber. The advantage of using 2D-array diode for QA is for the ability to perform absolute dose comparisons for hundreds of measurement positions using only a single beam delivery, as compared to the many multitudes of delivery repetitions necessary to perform absolute point measurements with a micro ionization chamber. ${ }^{9,10}$

Mainly 2D ARRAY is applicable in the case of Quality Assurance of high energy beams. For each application range separate analysis software like Multi Check or Verisoft is required. Software is available to do analytical comparison of measurements versus dose distributions calculated by a treatment planning system. This software is used to convert 2D dose maps from TPS to gray scale virtual films. It will be represented on the screen of the personal computer with the locations of the 729 ion chambers superimposed over the gray scale image. The measuring system consists of 2D ARRAY Seven29 
T10024, ARRAY INTERFACE T16026, RS232 Cable T22373/K6730 and Matrix Scan Software S080050. ${ }^{9}$

\section{Octavius phantom}

Octavius is an octagonal solid body phantom with an opening to insert the 2D ARRAY Seven29 for verification of dose distributions and absolute dose values of composite IMRT and VMAT plans. It was constructed for actual measurements with the Seven29 ion chamber array. It has a built-in cylindrically symmetric compensation cavity to correct for anisotropic behavior of the 2D ion chamber measurements. The 2D ARRAY Seven29 can be inserted into the Octavius Phantom for irradiation from different angles. The Octavius Phantom is made of polystyrene (water equivalent within $2 \%$ ). The Octavius Phantom of the following components. The Octavius phantom consists of a Top body T40051.1.007, Plate T40051.1.1004, Engraved lines for aligning with the laser, reclosable fastener, Accessory plate T40051.1.003 with inserted 2D-ARRAY Seven29 Base body "CT" T40051.1.010 for CT scan and Base body "LINAC" T40051.1.011. These two phantoms vary in different base bodies. The CT Phantom is about $2.5 \mathrm{Kg}$ heavier. The Patient Specific Quality Assurance with 2 phantoms, as the 2D ARRAY Seven 29 shows slightly reduced response if it is irradiated from the back side. In order to compensate this base body of the Octavius Phantom features a cavity. The base body of the OCTAVIUS CT Phantom does not feature a cavity. The Patient Plan will be calculated for the OCTAVIUS CT Phantom and will be delivered to the Octavius LINAC Phantom. In Octavius Linac phantom there is a C-shaped air cavity. Thus the reduced response of the 2D-ARRAY Seven29 irradiated from backside will be compensated. With its cubic detector and geometrically uniform matrix design, the octavius detector 729 provides significantly higher field coverage compared to other detectors. ${ }^{9-12}$

\section{Gamma analysis}

Gamma analysis is a method for comparing two dose distributions. Generally the dose distribution comparison is sub divided into regions of high and low dose gradients. In low gradient regions, the doses are compared directly with an acceptance tolerance placed on the difference between the two dose maps. In high dose gradient regions, a small spatial error, in either of the dose maps or small miss alignment results in large dose difference between the dose maps. Dose difference in the high dose gradient regions may therefore be relatively unimportant and the concept of Distance to Agreement (DTA) distribution is used to determine the acceptability of agreement. Dose difference in the high dose gradient regions may therefore be relatively unimportant and the concept of DTA distribution is used to determine the acceptability of agreement. The dose difference and DTA evaluations complement each other when used as determinants of agreement accuracy between the dose maps. The simultaneous use of DTA and a percent dose difference (DD) was proposed by Low et al. ${ }^{8}$ These parameters can help evaluate the agreement of the two distributions in terms of misalignment and difference, respectively. So in this study the various gamma index constraints which are a combination of particular DTA value with specific dose difference tolerance value were used. In this study gamma analysis was done for the whole arc. Gamma analysis of measured and TPS calculated fluence were cited in Figure 1.

\section{Verification plan}

45 RapidArc verification plans already delivered and analysed were selected for the study. Verification plans were created using
Eclipse treatment planning system version 10.0 (Varian Medical Systems, Palo Alto, CA) using AAA algorithm. It was delivered on Octavius phantom with 2D seven29 detector array (PTW, Freiburg, Germany) to study the response of the 2D seven29 detector array over a period of time. With the help of software Verisoft compared the dose distribution of measured and TPS calculated using the gamma-index method proposed by Low et al. ${ }^{8}$ The acceptance criteria of $3 \mathrm{~mm}$ for the distance to agreement (DTA) and dose difference tolerance level of $3 \%$ were chosen for analysis. Also the percentage of the evaluated dose points passing the gamma index was kept at a limit of greater than or equal to $95 \%$. Results of this pre-treatment quality assurance plans were compared with the results for the same plan done before stipulated period. Figure 2 shows the Verification Plan created in Eclipse Version 10.

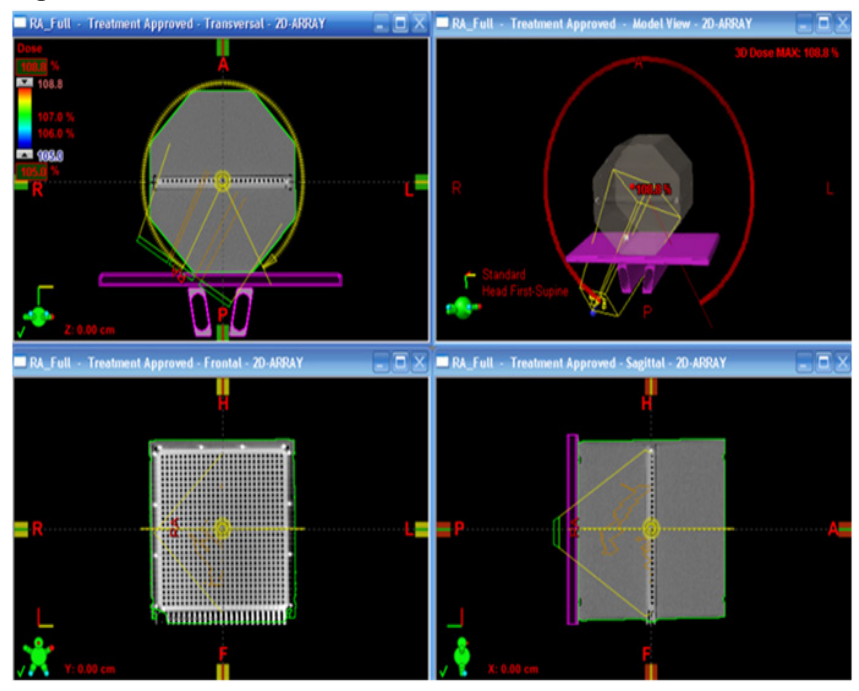

Figure I Verification plan created in eclipse 10 treatment planning system.

The verification plans created during the following periods were evaluated;

a. Response of 2D array to the plans before 2 Years.

b. Response of 2D array to the plans before 1.5 Years.

c. Response of 2D array to the plans before 1 Year.

\section{Results and discussion}

The average gamma pass percentage of present measurement and measurements before 2 years is 95.7 and 97.84 ; for 1.5 years is 97.02 and 98.28 ; for 1 year is 96.42 and 96.86 respectively. The result is cited in Table 1. Average response of gamma analysis for a period of 2 years, 1.5 years and 1 year is given in Figure 2 .

The plans which passed the gamma analysis (with percentage more than 95) were evaluated. Higher gamma passing criteria where observed for all analyzed plans, when analysis done before 2 years, 1.5 years and 1 year. Response of 2D array for the set of patients treated before 2years, 1.5years and 1 year Vs Present is given respectively in Table $1,2 \& 3$. Standard Deviation of gamma pass percentage before 2 years is 1.38 and for present measurement is 0.70 . Standard Deviation of gamma pass percentage before 1.5 years is 1.4 and for present measurement is 1.36. Standard Deviation of gamma pass percentage before 1 year is 0.97 and for present measurement is 1.18. Standard Deviation and percentage deviation of gamma pass percentage for present measurement Vs 2 years, 1.5 years and 1 year were given graphically in Figure 4 \& Figure 5 respectively. 


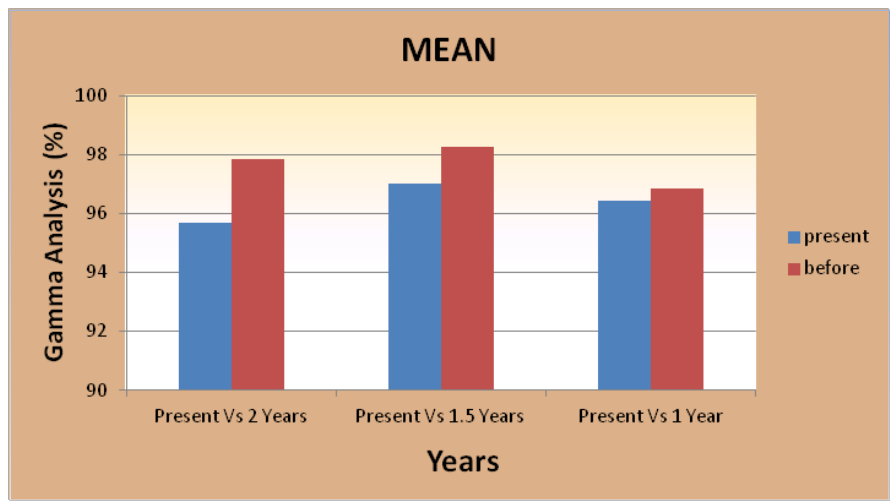

Figure 2 Mean response of 2D array of a set of patients treated before Vs present.

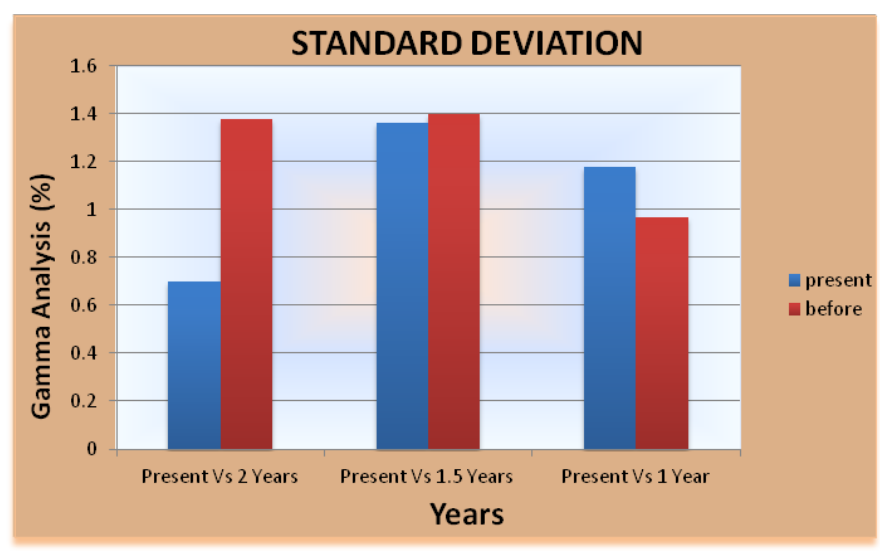

Figure 3 Standard deviation of gamma pass percentage for present measurement $V s 2$ years, I.5 years and I year.

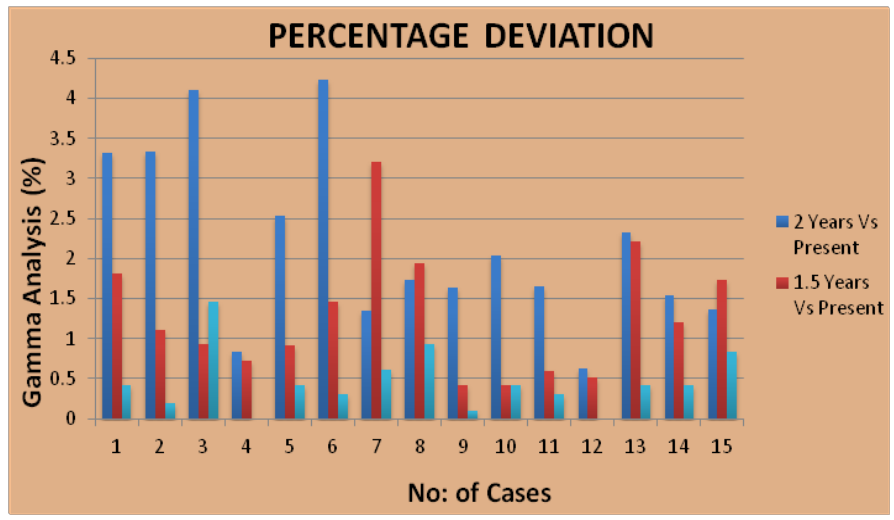

Figure 4 Percentage deviation of the gamma analysis for present measurement Vs 2 years, I. 5 years and I year.

This study shows that the response of $2 \mathrm{D}$ array reduces with period. The reason for reducing the response of the chamber may because of damage to any one of the chamber in the array. Sensitivity is also another factor that affects the response. Sensitivity decreases if the chamber gets damaged. If the radiation affects the circuit region in the 2D array it can damage the array. So, that individualized check of each ionization chamber inside the array is needed.
Table I Response of 2D array for the set of patients treated before 2years Vs present

\begin{tabular}{llll}
\hline \multicolumn{4}{l}{ Response of 2D array to the plans before 2 years } \\
\hline \multicolumn{3}{l}{ Gamma analysis } \\
Patient No. & before 2 year & Present & \%Variation \\
1 & 99.5 & 96.2 & 3.32 \\
2 & 98.8 & 95.5 & 3.34 \\
3 & 99.7 & 95.6 & 4.11 \\
4 & 96.2 & 95.4 & 0.83 \\
5 & 99 & 96.5 & 2.53 \\
6 & 99.3 & 95.1 & 4.23 \\
7 & 97 & 95.7 & 1.34 \\
8 & 97.9 & 96.2 & 1.74 \\
9 & 97.4 & 95.8 & 1.64 \\
10 & 98.5 & 96.5 & 2.03 \\
11 & 96.7 & 95.1 & 1.65 \\
12 & 96.4 & 95.8 & 0.62 \\
13 & 98.8 & 96.5 & 2.33 \\
14 & 97.3 & 95.8 & 1.54 \\
15 & 95.1 & 93.8 & 1.37 \\
\hline
\end{tabular}

Table 2 Response of $2 \mathrm{D}$ array for the of set patients treated before I.5Years Vs present

\begin{tabular}{llll}
\multicolumn{4}{l}{ Response of 2D array to the plans before I.5 years } \\
\hline \multicolumn{4}{l}{ Gamma analysis-3mm, 3\% } \\
Pt. No: & before I.5yr & Present & $\%$ V \\
I & 99.3 & 97.5 & 1.81 \\
2 & 100 & 98.9 & 1.1 \\
3 & 97.1 & 96.2 & 0.93 \\
4 & 96.2 & 95.5 & 0.73 \\
5 & 97.8 & 96.9 & 0.92 \\
6 & 95.7 & 94.3 & 1.46 \\
7 & 99.7 & 96.5 & 3.21 \\
8 & 97.7 & 95.8 & 1.94 \\
9 & 98.5 & 98.1 & 0.41 \\
10 & 98.1 & 97.7 & 0.41 \\
II & 99.6 & 99 & 0.6 \\
12 & 97 & 96.5 & 0.52 \\
13 & 99.7 & 97.5 & 2.21 \\
14 & 100 & 98.8 & 1.2 \\
15 & 97.8 & 96.1 & 1.74 \\
\hline
\end{tabular}


Table 3 Response of 2D array for the set of patients treated before I year Vs present

\begin{tabular}{|c|c|c|c|}
\hline \multicolumn{4}{|c|}{ Response of 2D array to the plans before I year } \\
\hline \multicolumn{4}{|c|}{ Gamma analysis-3mm, 3\% } \\
\hline Pt. No: & before Iyr & present & $\% \mathbf{V}$ \\
\hline 1 & 95.6 & 95.2 & 0.42 \\
\hline 2 & 98.2 & 98 & 0.2 \\
\hline 3 & 95.9 & 94.5 & 1.46 \\
\hline 4 & 97.1 & 97.1 & 0 \\
\hline 5 & 96.6 & 96.2 & 0.41 \\
\hline 6 & 97.3 & 97 & 0.31 \\
\hline 7 & 98.4 & 97.8 & 0.61 \\
\hline 8 & 96.4 & 95.5 & 0.93 \\
\hline 9 & 96.6 & 96.5 & 0.1 \\
\hline 10 & 95.5 & 95.1 & 0.42 \\
\hline 11 & 97.8 & 97.5 & 0.31 \\
\hline 12 & 98 & 98 & 0 \\
\hline 13 & 97.5 & 97.1 & 0.41 \\
\hline 14 & 96.2 & 95.8 & 0.42 \\
\hline 15 & 95.8 & 95 & 0.84 \\
\hline
\end{tabular}

\section{Conclusion}

It is found that $2 \mathrm{D}$ array shows the reduced response against radiation detection over a period of years. An onsite calibration of the instrument is recommended before the measurements. A dose correction factor is to be applied if the radiation response and efficiency of the array on radiation detection is poor.

\section{Financial disclosure statement}

On behalf of all the contributors I hereby assure that there is no financial aid for this proposed research work.

\section{Conflicts of interest}

On behalf of all the contributors I will act and guarantor and will correspond with the journal from this point onward. Also I hereby assure that there is no conflict of interest, regarding this article.

\section{References}

1. Otto K. Volumetric modulated arc therapy : IMRT in a single gantry arc. Med Phys. 2008;35(1):310-317.

2. Shueng P, Chong N, Lee H, et al. Feasibility of using a 2D Array Detector to Verify Composite IMRT Delivery for Helical Tomotherapy and Linac. Int J Rad Onc Biol Phys. 2008;72(1):S675-S675.

3. Dong L, Antolak J, Salepour M, et al. Patient-specific point dose measurement for IMRT monitor unit verification. Int J Radiat Oncol Biol Phys. 2003;56(3):867-877.

4. SE Burch, KJ Clark. The use of radiographic film for linear accelerator sterotactic radiosurgical dosimetry. Med Phys. 1999;26(10);2144-2150.

5. Saini AS, Zhu TC. Energy dependence of commercially available diode detectors for in-vivo dosimetry. Med Phys. 2007; 34(5):1704-1711.

6. Yu CX. Intensity-Modulated arc therapy with dynamic multileaf collimation: An alternative to tomotherapy. Phys Med Biol. 1995;40(9):1435-1449.

7. De Gersem W, Claus F, De Wagter C, et al. Leaf position optimization for step-and-shoot IMRT. Int J Radiat Oncol Biol Phys. 2002;29(5):10071018.

8. Daniel A Low. Gamma Dose Distribution Evaluation Tool. Journal of Physics: Conference Series. 2010;250.

9. Spezi E, Angelini AL, Romani F, et al. Characterization of a 2D ion Chamber ARRAY for the verification of radiotherapy treatments. Phys Med Biol. 2005;50(14):3361-3373.

10. VanEsch A, Clermon C, Devillers M, et al. On- line Quality Assurance of rotational radiotherapy treatment delivery by means of a $2 \mathrm{D}$ ion chamber array and the OCTAVIUS Phantom. Med Phys. 34(10);3825-3837.

11. Jrsinic PA, Nelms BE. A 2-D diode array and analysis software for verification of intensity modulated radiation therapy delivery. Med Phys. 2003;30(5):870-879.

12. Bedford JL, Lee YK, Wai P, et al. Evaluation of the Delta(4) phantom for IMRT and VMAT verification. Phys Med Biol. 2009;54(9):N167-176.

\section{Acknowledgements}

None. 